\title{
Differential Effects of C- and N-Terminal Substance P Metabolites on the Release of Amino Acid Neurotransmitters from the Spinal Cord: Potential Role in Nociception
}

\author{
Stephen R. Skilling, David H. Smullin, and Alice A. Larson \\ Department of Veterinary Biology, University of Minnesota, St. Paul, Minnesota 55108
}

Extensive evidence implicates Substance $P$ [SP(1-11)] as a primary afferent neurotransmitter or modulator of nociceptive information, and there is increasing evidence that the excitatory amino acids aspartate (Asp) and glutamate (Glu) may also act as nociceptive neurotransmitters. We have previously demonstrated that nociceptive stimulation (metatarsal injection of formalin) caused a tetrodotoxin (TTX)sensitive release of Asp and a TTX-insensitive release of Glu from the dorsal spinal cord. We have also shown release of Asp and Glu following the direct infusion of SP(1-11), suggesting that formalin-induced Asp or Glu changes could be secondary to an initial release of SP(1-11). In contrast to nociception, pretreatment with TTX, reported here, had no effect on the SP(1-11)-induced release of Asp, suggesting a presynaptic mechanism. Behavioral experiments, in both our laboratory, and others, now suggest that the $\mathrm{N}$-terminal products of SP metabolism play a distinct role in the modulation of SP(1-11) nociception, possibly through an interaction with an opiate receptor. To test the hypothesis that $\mathbf{N}$ - and $\mathbf{C}$-terminal fragments of SP produce opposite effects on biochemical events potentially involved in nociception, we compared the effects of infusion of the N-terminal metabolite SP(1-7) and the C-terminal metabolite SP(511) on changes in the ECF concentration of amino acids in the spinal cord as a measure of their apparent release, using microdialysis. Intradiaylsate infusion of SP(5-11) increased the release of Asp, Glu, asparagine (Asn), glycine (Gly), and taurine (Tau). The changes in Asp, Glu, and Tau were similar in direction and magnitude to changes produced by SP(111) or formalin injection, further supporting the hypothesis that the $\mathrm{C}$-terminal is responsible for the nociceptive effects of SP(1-11). In contrast, infusion of SP(1-7) significantly decreased the release of Asn, Tau, Glu, and Gly. This inhibition of amino acid release is consistent with the hypothesis that N-terminal metabolites produce opposite effects to those of C-terminal metabolites of SP(1-11). The decreases in Glu,

\footnotetext{
Received May 18, 1989; revised Sept. 29, 1989; accepted Nov. 8, 1989.

The use and care of animals in this study was performed in accordance with the guidelines of the Minnesota Animal Care and Use Committee and those prepared by the Committee on Care and Use of L aboratory Animals of the Institute of Laboratory Animal Resources, National Research Council [DHEW Publication no. (NIH) 78-23, revised 1978]. This research was supported by U.S. Public Health Service Grants DA04090, DA04190, and DA00124 to A.A.L., CA01342 to S.R.S., and DA07234 to D.H.S.

Correspondence should be addressed to Dr. Stephen R. Skilling, Department of Veterinary Biology, 295 Animal Science/Veterinary Medicine Building, University of Minnesota, 1988 Fitch Ave., St. Paul, MN 55108.

Copyright (C) 1990 Society for Neuroscience 0270-6474/90/041309-10\$02.00/0
}

Asn, Gly, and Tau following SP(1-7) infusion were significantly reduced by i.p. or intradialysate naloxone. Systemic naloxone had no significant effects on the SP(5-11)-induced amino acid changes; however, it did inhibit the SP(1-11)induced increase in Asp and Glu. Intradialysate naloxone had no effect on the SP(1-11)-induced increases. These results support the hypothesis that $\mathbf{N}$-terminal fragments of SP, generated by SP(1-11) metabolism in vivo, may be responsible for the naloxone-reversible analgesic effects of SP(1-11) while the nociceptive actions of SP(1-11) may be mediated through its C-terminal end.

Intravenous or intracerebral Substance $\mathrm{P}$ [SP(1-11)] produces both hyperalgesia and, at low doses, a naloxone-sensitive analgesia (Stewart et al., 1976; Frederickson et al., 1978; Oehme et al., 1980). It has been hypothesized that these differential effects of SP(1-11) in the spinal cord are mediated through 2 distinct receptors, a $\mathrm{C}$-terminal receptor that mediates the nociceptive actions of $\mathrm{SP}(1-11)$ and an $\mathrm{N}$-terminal receptor that mediates the antinociceptive effects (Stewart et al., 1982). While SP(1-11) may be the primary ligand for these receptors, C- and N-terminal fragments of SP degradation may also act as endogenous ligands. An enzymatic pathway for the degradation of SP(1-11) in situ has been shown (Lee et al., 1981; Nyberg et al., 1984). Sakurada and colleagues (1985) have measured SP(17) and C-terminal fragments in homogenized rat brain and spinal cord, and recently, endogenous concentrations of the N-terminal fragments SP(1-4), SP(1-7), and SP(1-9) and the C-terminal fragments $\mathrm{SP}(2-11)$ and $\mathrm{SP}(5-11)$ have been measured in mouse spinal cords in vitro (Igwe et al., 1988a). While an active uptake system for the C-terminal fragment SP(5-11) has been demonstrated that would theoretically maintain low extracellular concentrations of this metabolite following SP(111) release, no uptake system has been found for intact SP (Nakata et al., 1981). If an uptake system for N-terminal SP fragments is also not present, $\operatorname{SP}(1-7)$ could accumulate during $\mathrm{SP}(1-11)$ release and may be responsible for $\mathrm{SP}(1-11)$ antinociception through an interaction with $\mathrm{N}$-terminal receptors. In support of this hypothesis, intraperitoneal injection of the N-terminal metabolite $\mathrm{SP}(1-7)$ produccs a dosc-dependent antinociception in both the tail flick and hot plate assay that is not seen following injection of SP C-terminal fragments (Hall and Stewart, 1983; Cridland and Henry, 1988).

Further evidence for an effect of the accumulation of N-terminal SP fragments on SP behavior has been shown using the biting and scratching behavioral response seen following intrathecal injections of SP(1-11) in mice. Intrathecal SP(5-11) 
produces biting and scratching similar to SP(1-11) while SP(17) produces a naloxone-reversible inhibition of the behavior induced by simultaneous injection of SP(1-11) or SP(5-11) (Igwe et al., 1988b). While repeated intrathecal injections of $\operatorname{SP}(1-11)$ result in a naloxone-sensitive desensitization to biting and scratching, presumably through an accumulation of $\mathrm{N}$-terminal fragments (Larson, 1988), this desensitization is not seen following repeated injections of SP(5-11) (Igwe et al., 1988b). Repeated injections of SP(1-7), on the other hand, result in desensitization to the behavior produced by a subsequent injection of SP(1-11) (Larson and Sun, personal communication, 1989). Prevention of $\mathrm{N}$-terminal production through the inhibition of SP(1-11) degradative enzymes blocks the development of desensitization to SP(1-11)-induced behavior (Igwe et al., 1988b).

While $\mathrm{SP}(1-11)$ has been proposed as a primary afferent neurotransmitter, the slow time course of dorsal horn neuron depolarization following iontophoretic application of SP(1-11) and the lack of effect on basal pain response following desensitization to $\mathrm{SP}(1-11)$-induced hyperalgesia have prompted some researchers to suggest that $\mathrm{SP}(1-11)$ is a modulator of a faster neurotransmitter system (Henry, 1976; Sweeney and Sawynok, 1986). The excitatory amino acids (EAAs) glutamate (Glu) and aspartate (Asp) have been suggested to be primary afferent nociceptive transmitters capable of this fast transmission (Schneider and Perl, 1985). Evidence exists for an interaction between SP(1-11) and EAAs at the spinal level: (1) SP(1-11)- and Glulike immunoreactivity are colocalized in dorsal root ganglion neurons in the rat and monkey (Battaglia and Rustioni, 1988); (2) $\mathrm{SP}(1-11)$ causes the release of Glu from hemisected dorsal spinal cords in both frogs and newborn rats (Kawagoe et al., $1985,1986)$; and (3) we have shown that either SP(1-11) or nociceptive stimulation produces a significant increase in the apparent release of Asp and Glu in the dorsal spinal cord of awake rats (Skilling et al., 1988; Smullin et al., 1990).

The goal of the present study was to determine whether the enhanced rclcase of EAAs in the spinal cord following SP(1-11) is mediated by $\mathrm{C}$ - or $\mathrm{N}$-terminal portions of $\mathrm{SP}(1-11)$. To accomplish this we used in vivo microdialysis of the spinal cord of conscious freely moving rats to measure the effect of exogenous application of the N-terminal metabolite SP(1-7) and the C-terminal metabolite $\operatorname{SP}(5-11)$ on amino acid concentrations in the dorsal spinal cord extracellular fluid as a measure of their apparent release. We have previously shown the success of this model for measuring changes in amino acid release following chemical and nociceptive stimulation (Skilling et al., 1988) and following SP(1-11) infusion (Smullin et al., 1990). Using the sodium channel blocker tetrodotoxin (TTX), we have also demonstrated the involvement of sodium channels in nociceptiveinduced Asp release.

In the present study we have again used TTX to characterize the involvement of sodium channels in the SP(1-11)-induced amino acid release. In this paper, we also report neurochemical evidence of a unique opposing action of $\mathrm{N}$ - and C-terminal SP fragments on the release of putative amino acid neurotransmitters. These results are discussed in terms of a potentially important analgesic role for endogenously produced N-terminal SP metabolites.

\section{Materials and Methods}

Microdialysis. Male Sprague-Dawley rats (275-300 gm) were anesthetized with sodium pentobarbitol $(50 \mathrm{mg} / \mathrm{kg}$, i.p., Veterinary Laboratories, Inc.) and implanted with a microdialysis fiber (200- $\mu \mathrm{m}$ diameter,
50,000 MW cutoff, Amicon Vitafiber I) through the dorsal spinal cord as described previously (Skilling et al., 1988) with one modification. Prior to implantation of the cannula, the stylette was removed and one end of the cannula was glued with epoxy to an 8-mm-long, $0.15-\mathrm{mm}$ diameter minutien pin (Fine Science Tools). The pin was pushed transversally through the cord and the cannula pulled into place. The pin was then cut off of the cannula before the cannula was connected to PE 10 tubing.

Following a 24-hr recovery period, the animals were evaluated for any signs of limb paralysis or impaired movement. No impairment was observed in any of the animals in this study and all animals appeared to behave normally. The dialysis system was attached to a peristaltic pump (Gilson Minipuls 3) and perfused with Ringer's solution (147 mM $\mathrm{NaCl}, 4.0 \mathrm{~mm} \mathrm{KCl}, 2.2 \mathrm{mM} \mathrm{CaCl}_{2}$ ) at $5-6 \mu \mathrm{l} / \mathrm{min}$ for $90 \mathrm{~min}$ to establish a diffusion equilibrium. Samples were collected at $10-\mathrm{min}$ intervals in polypropylene tubes and maintained at $5^{\circ} \mathrm{C}$ until analyzed for amino acids within $12 \mathrm{hr}$ by high-performance liquid chromatography (HPLC) as described by Skilling et al. (1988). Animals were maintained at $22^{\circ} \mathrm{C}$ with 12-hr light/dark cycles and were provided with food and water ad libitum.

Following each experiment, the animals were killed and the cannula injected with new methylene blue dye. The spinal cord was then removed and fixed for gross histological confirmation of cannula placement. Only animals with cannulas located below lamina I and above the central canal were included in this study. In addition, animals were not included in this study if evidence of dye leakage into the cerebral spinal fluid was present.

Three samples were collected for determination of basal concentrations of amino acids. Administration of SP(1-11) (Bachem), SP(1-7) (Bachem), or SP(5-11) (Bachem) was made by the addition of $1 \mathrm{~mm}$ $\mathrm{SP}(1-11), 1 \mathrm{~mm} \mathrm{SP}(1-7)$, or $0.5 \mathrm{~mm} \mathrm{SP}(5-11)$ to the Ringer's solution for $60 \mathrm{~min}$. This concentration of SP(1-11) represents the minimum effective concentration, while the concentration of SP(1-7) was chosen to be equimolar to the SP(1-11) given. Due to the poor solubility of 1 mM SP(5-11) in Ringer's solution, we used a concentration of $0.5 \mathrm{~mm}$. In vitro experiments, using known concentrations of peptide standards at $37^{\circ} \mathrm{C}$, have shown a linear relationship between peptide concentration in the standards and recovery of each peptide in the dialysis solution as measured by HPLC. Assuming that the diffusion of these peptides across the dialysis membrane is approximately equal in both directions, the dialysate concentrations of the peptides used would result in an estimated maximum diffusion of $1.0 \mathrm{nmol}$ of $\mathrm{SP}(1-11)$ and $\mathrm{SP}(1-7)$ and $0.25 \mathrm{nmol}$ of SP(5-11) into the ECF during each 10-min perfusion period. Intradialysate naloxone was administered by the addition of 31 mM naloxone ( $\mathrm{Du}$ Pont Pharmaceuticals) to the dialysate one sample prior to and throughout the peptide administration. This concentration of naloxone represents the estimated level required to achieve a delivery of $1 \mu \mathrm{g} / \mathrm{min}$ [a dose comparable to that given i.t. in mice in previous studies (Larson, 1988)] into the ECF. Systemic naloxone $(5 \mathrm{mg} / \mathrm{kg}$ ) was administered intraperitoneally at 30 -min intervals beginning $10 \mathrm{~min}$ before peptide administration. Ten-minute samples were collected throughout the course of the experiments described above.

To examine the role of sodium channels in SP(1-11)-induced Asp and Glu release, animals were perfused with Ringer's solution and 3 control samples were taken for determination of basal release. SP(111 ), at a concentration of $1 \mathrm{~mm}$, was then perfused through the animal for $20 \mathrm{~min}$ followed by Ringer's solution for $30 \mathrm{~min}$. The animals were then perfused with $10 \mu \mathrm{M}$ TTX for one 10-min period followed again by $1 \mathrm{~mm} \mathrm{SP}(1-11)$ for $20 \mathrm{~min}$ and then Ringer's solution for $30 \mathrm{~min}$.

For statistical analysis of peptide effects, the data were converted into ECF concentration $(\mu \mathrm{M})$ using in vitro calibration. Calibration was performed at $37^{\circ} \mathrm{C}$ by placing dialysis cannulas into vials containing amino acid standards in Ringer's solution. Dialysis recovery of amino acids was linear and an appropriate regression equation for each amino acid was determined. The mean of the first 3 samples following treatment was compared to the mean of the 3 samples prior to treatment using paired $t$-tests. For testing naloxone effects, the data were further transformed into percent of basal concentration and the means of the first 3 samples following treatment were analyzed across treatments using analysis of variance (ANOVA) with Scheffe's $F$-test for comparison of individual means.

For comparison of TTX effects, the data were again converted into ECF concentration $(\mu \mathrm{M})$ using in vitro calibration and the maximal release occurring during the 20 -min $\operatorname{SP}(1-11)$ infusion was compared to the maximal release occurring during TTX followed by SP(1-11) using 
Table 1. Effects of Substance $P$ and metabolites on dorsal spinal cord amino acid release

Amino acid (percent of basal concentration \pm SEM) ${ }^{a}$

\begin{tabular}{|c|c|c|c|c|c|}
\hline Peptide & Asp & Glu & Asn & Gly & Tau \\
\hline Substance $P(1-11)$ & $141.5 \pm 4.1^{b}$ & $130.7 \pm 8.3^{b}$ & $101.0 \pm 2.7$ & $115.9 \pm 3.8^{b}$ & $171.3 \pm 30.6$ \\
\hline + systemic naloxone & $113.0 \pm 4.6^{c}$ & $104.8 \pm 4.2^{d}$ & $88.4 \pm 3.4$ & $98.2 \pm 2.9$ & $229.3 \pm 33.7$ \\
\hline+ intradialysate naloxone & $142.9 \pm 5.4$ & $129.3 \pm 5.8$ & $110.7 \pm 9.1$ & $235.8 \pm 80.3$ & $213.0 \pm 38.7$ \\
\hline Substance $P(5-11)$ & $182.5 \pm 20.6^{b}$ & $231.6 \pm 38.5^{b}$ & $120.2 \pm 2.7^{b}$ & $183.5 \pm 17.2^{b}$ & $360.9 \pm 81.8^{b}$ \\
\hline + systemic naloxone & $210.5 \pm 53.4$ & $187.3 \pm 38.1$ & $118.0 \pm 14.0$ & $158.6 \pm 19.9$ & $267.0 \pm 64.1$ \\
\hline Substance $P(1-7)$ & $133.2 \pm 22.8$ & $66.2 \pm 12.3^{e}$ & $42.6 \pm 4.9^{b}$ & $50.9 \pm 14.7^{e}$ & $31.7 \pm 11.8^{b}$ \\
\hline + systemic naloxone & $106.2 \pm 5.7$ & $100.2 \pm 9.4^{d}$ & $72.9 \pm 2.8^{d}$ & $121.9 \pm 24.2^{d}$ & $106.3 \pm 16.5^{d}$ \\
\hline + intradialysate naloxone & $117.0 \pm 17.4$ & $132.4 \pm 20.5^{d}$ & $90.0 \pm 9.3^{c}$ & $107.3 \pm 5.4^{d}$ & $156.4 \pm 22.5^{c}$ \\
\hline
\end{tabular}

${ }^{a}$ Based on the mean ECF concentration recovered during the first 310 -min samples folowing peptide administration as determined by transformation of dialysate concentrations using in vitro calibration.

${ }^{b}$ Significantly different from basal concentration at $p<0.01$ using Student's paired $t$-test.

Significantly different from peptide effect on concentration in absence of naloxone at $p<0.01$ using ANOVA.

${ }^{d}$ Significantly different from peptide effect on concentration in absence of naloxone at $p<0.05$ using ANOVA.

'Significantly different from basal concentration at $p<0.05$ using Student's paired $t$-test.

paired $t$-tests. Previous studies with control animals showed no significant difference between the response to the first and second challenge with SP(1-11) in the absence of TTX and no effect of TTX by itself.

\section{Results}

The mean amounts of amino acids recovered in the first 310 min dialysate samples collected following continuous peptide infusion were measured and transformed into extracellular concentrations using in vitro calibration of the dialysis cannula. These treatment means were subsequently divided by the mean extracellular concentration of the 310 -min dialysate samples collected prior to peptide infusion to obtain the percent of basal concentration values \pm SEM presented in Table 1 .

Infusion of $0.5 \mathrm{~mm} \mathrm{SP(5-11)} \mathrm{via} \mathrm{the} \mathrm{dialysate} \mathrm{significantly}$ increased the release of Asp (82\%), Glu (132\%), asparagine (Asn) $(21 \%)$, glycine (Gly) (83\%), and taurine (Tau) $(261 \%)(p<0.01)$ (Table 1). Maximal changes in the release of these amino acids occurred during the first $10 \mathrm{~min}$ of SP(5-11) infusion and, with the exception of Asn, the amino acid release declined over the next $50 \mathrm{~min}$, despite continuous peptide infusion (Figs. $1 B-5 B$ ). This is in contrast to the maintained elevations in Asp, Glu and Tau release seen following continuous SP(1-11) infusion (Figs. $1 A-5 A$ ). While we have previously reported a transient (less than $10 \mathrm{~min}$ ), caudally directed biting and scratching behavior in animals following continuous $\mathrm{SP}(1-11)$ infusion, this behavior was not observed in animals following SP(5-11).

Infusion of $1 \mathrm{~mm}$ SP(1-7) not only failed to increase Asp, but significantly decreased the release of Asn (57\%) and Tau (68\%) $(p<0.01)$ and Glu (34\%) and Gly (49\%) $(p<0.05)$ (Table 1$)$. The release of Asn, Glu, and Gly continued to decrease over the entire $\mathrm{SP}(1-7)$ infusion period, while the release of Tau reached a minimal value at $30 \mathrm{~min}$ with partial recovery over the next $30 \mathrm{~min}$ of peptide infusion (Figs. $1(-5 C)$ ). No readily apparent behavioral responses were observed during $\operatorname{SP}(1-7)$ infusion.

Systemic i.p. injection of $5 \mathrm{mg} / \mathrm{kg}$ of naloxone $10 \mathrm{~min}$ prior to peptide administration had no effect on the basal release of amino acids when given alone and also failed to affect the $\operatorname{SP}(5-$ 11)-induced increases in amino acids. Prior treatment with systemic naloxone did, however, significantly reduce the change in Asn, Gly, and Tau $(p<0.05)$ observed following SP(1-7) (Table 1). Systemic naloxone also reduced the elevations in Asp $(p<$
$0.01)$ and Glu $(p<0.05)$ release observed following $\operatorname{SP}(1-11)$ (Table 1). Intradialysate infusion of $31 \mathrm{~mm}$ naloxone alone had no effect on amino acid release but did inhibit SP(1-7)-induced changes in Asn and Tau $(p<0.01)$ and Glu and Gly $(p<0.05)$. Intradialysate naloxone had no effect on the SP(1-11)-induced increases (Table 1).

Pretreatment with $10 \mu \mathrm{M}$ TTX had no effect on the release of Asp or Gly induced by SP(1-11) (Table 2). In contrast, the SP(111 )-induced release of Glu and Tau was $200 \%$ greater following pretreatment with TTX (Table 2). Behaviorally, tetrodotoxin pretreatment did not alter the intensity or occurrence of caudally dirceted biting and licking seen following SP(1-11). The animals did, however, appear to be more hyperresponsive to external noise than animals receiving $\mathrm{SP}(1-11)$ alone.

\section{Discussion}

In this study, the increases in amino acid release in the dorsal spinal cord ECF following the C-terminal fragment SP(5-11) support the hypothesis that the C-terminal end of the $\operatorname{SP}(1-11)$ molecule is responsible for the amino acid changes seen following SP(1-11). The N-terminal fragment SP(1-7), on the other hand, produces decreases in amino acid release, supporting the hypothesis that $\mathrm{N}$-terminal fragments of SP act as neuromodulators of spinal transmission working in opposition to $\mathrm{SP}(1-11)$ or its C-terminal peptide fragments. These opposing actions of SP fragments on amino acid release may explain the dual action

Table 2. Effect of TTX on SP(1-11)-induced release of Glu and Asp (percent of basal concentration \pm SEM) ${ }^{a}$

\begin{tabular}{llc} 
Amino acid & SP(1-11) & SP(1-11) plus TTX \\
\hline Asp & $299.3 \pm 54.8$ & $465.0 \pm 88.6$ \\
Glu & $246.1 \pm 12.6$ & $685.5 \pm 150.4^{b}$ \\
Asn & $119.5 \pm 7.9$ & $147.5 \pm 13.3$ \\
Gly & $175.1 \pm 22.0$ & $255.0 \pm 38.6$ \\
Tau & $447.5 \pm 62.5$ & $1088.4 \pm 112.3^{b}$
\end{tabular}

${ }^{a}$ Based on the mean ECF concentration recovered during the first $310-\mathrm{min}$ samples following peptide administration with or without TTX as determined by transformation of dialysate concentrations using in vitro calibration.

${ }^{b}$ Significantly different from SP(1-11) effect on concentration in absence of TTX at $p<0.025$ using paired $t$-tests. 
Asp
Figure 1. Time course of the changes in the concentration of Asp following infusion, starting at time zero, of $(A)$ $1.0 \mathrm{~mm}$ substance $\mathrm{P},(B) 0.5 \mathrm{~mm}$ substance $P(5-11)$, and $(C) 1.0 \mathrm{~mm}$ substance $P(1-7)$ through a $0.2-\mathrm{mm}$ dialysis cannula (Amicon Vitafiber I) implanted in the dorsal lumbar spinal cord of rats. Animals in group 1 (open $b o x)$ received peptide alone. Animals in group 2 (closed box) were given $5 \mathrm{mg} /$ $\mathrm{kg}$ of naloxone i.p. at $10 \mathrm{~min}$ prior to peptide infusion and $30 \mathrm{~min}$ following the first injection. Animals in group 3 (open circle) were given $31 \mathrm{~mm}$ naloxone via the dialysis cannula beginning $10 \mathrm{~min}$ prior to and continuing throughout peptide infusion. Experiments were run using conscious animals, $24 \mathrm{hr}$ following implantation of the cannula. Ringer's solution with and without peptide was perfused through the cannula at $5 \mu \mathrm{l} / \mathrm{min}$. Samples were at $5^{\circ} \mathrm{C}$ until analyzed for amino acids using HPLC. Concentrations recovered in the dialysis samples were converted to extracellular fluid concentration using in vitro calibration curves. Values are expressed as a percent of the basal concentration, which was determined as the mean extracellular fluid concentration of the first $310-\mathrm{min}$ samples following an initial $60-$ min perfusion to establish dialysis equilibrium. $n=5-6$ animals per group. collected every $10 \mathrm{~min}$ and maintained

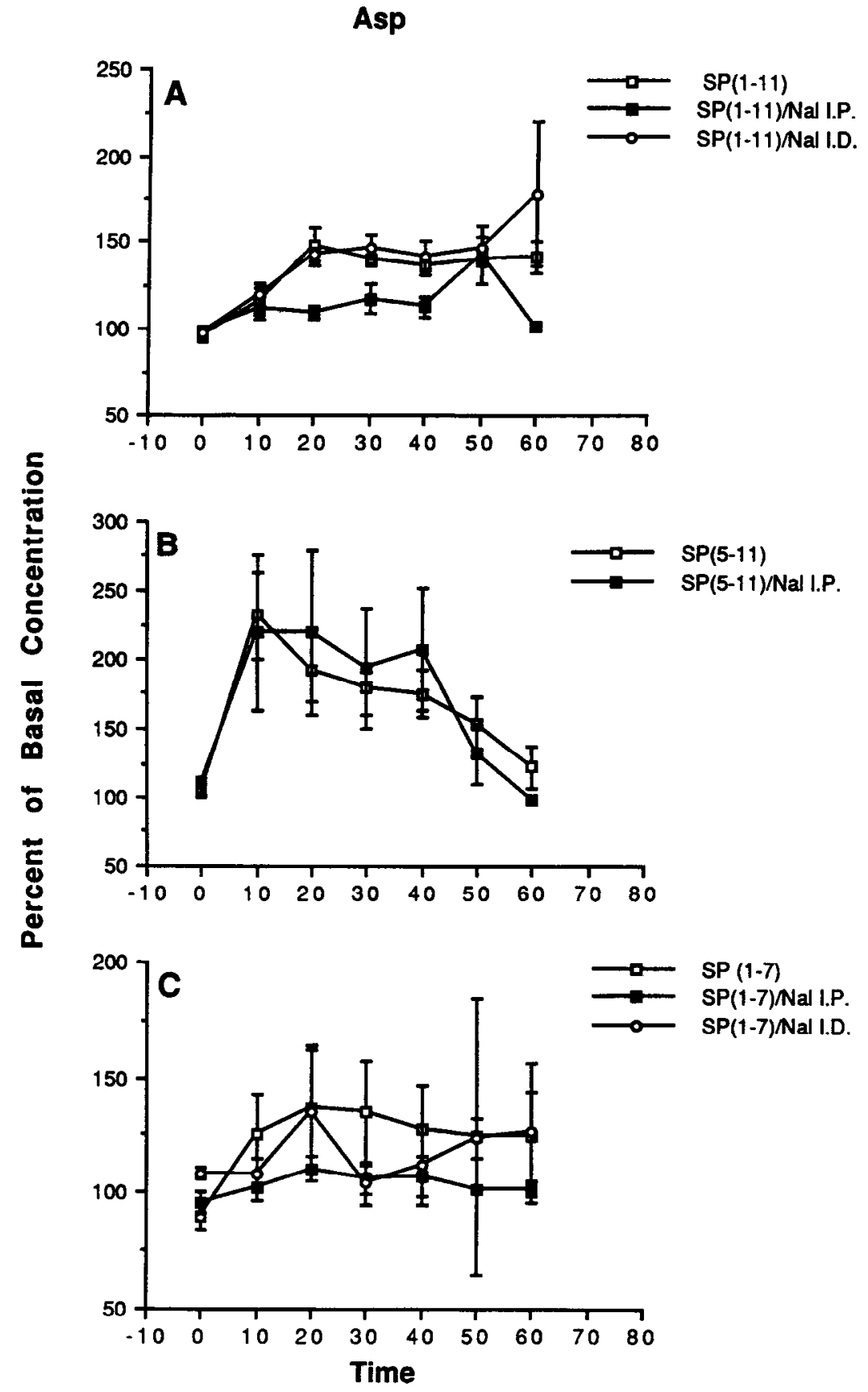

of SP with respect to pain. In contrast to the hyperalgesic effect most frequently associated with SP administration (Hayes and Tyers, 1979; Oehme et al., 1980), a delayed analgesic effect occurring 15-30 min following intraperitoneal or intracerebroventricular injection has been reported (Stewart et al., 1976; Frederickson et al., 1978; Oehme et al., 1980). One hypothesis for the delayed analgesia was that it was due to the release of endogenous opiates as the antinociceptive effect was reversed by naloxone. An alternate hypothesis proposed that $\mathrm{SP}(1-11)$ was metabolized to an active $\mathrm{N}$-terminal fragment (Stewart et al., 1982; Cridland and Henry, 1988). In support of the latter hypothesis, intrathecal injection of SP(1-7) produces antinociception with a similar restricted dose range to that seen following intrathecal SP(1-11), but with a shorter onset of action, as would be predicted given that $\mathrm{SP}(1-7)$ would not require prior metabolism to an active form (Cridland and Henry, 1988).

Both the analgesia and the decreases in amino acids observed following SP(1-7) were antagonized by naloxone, supporting an involvement of opiate receptors in these effects. We have recently shown that $\mathrm{SP}(1-7)$ can directly bind to mu-1 opiate receptors (Krummins et al., 1989). The decreases in amino acid release following SP(1-7), therefore, are likely mediated through a mu-1 receptor and antagonism by naloxone involves displacement of SP(1-7) from this receptor. Antagonism occurred following both systemic and intradialysate naloxone application. Assuming that the diffusion of naloxone from the dialysis cannula is limited within the spinal region being perfused, these results support a spinal site of action. 


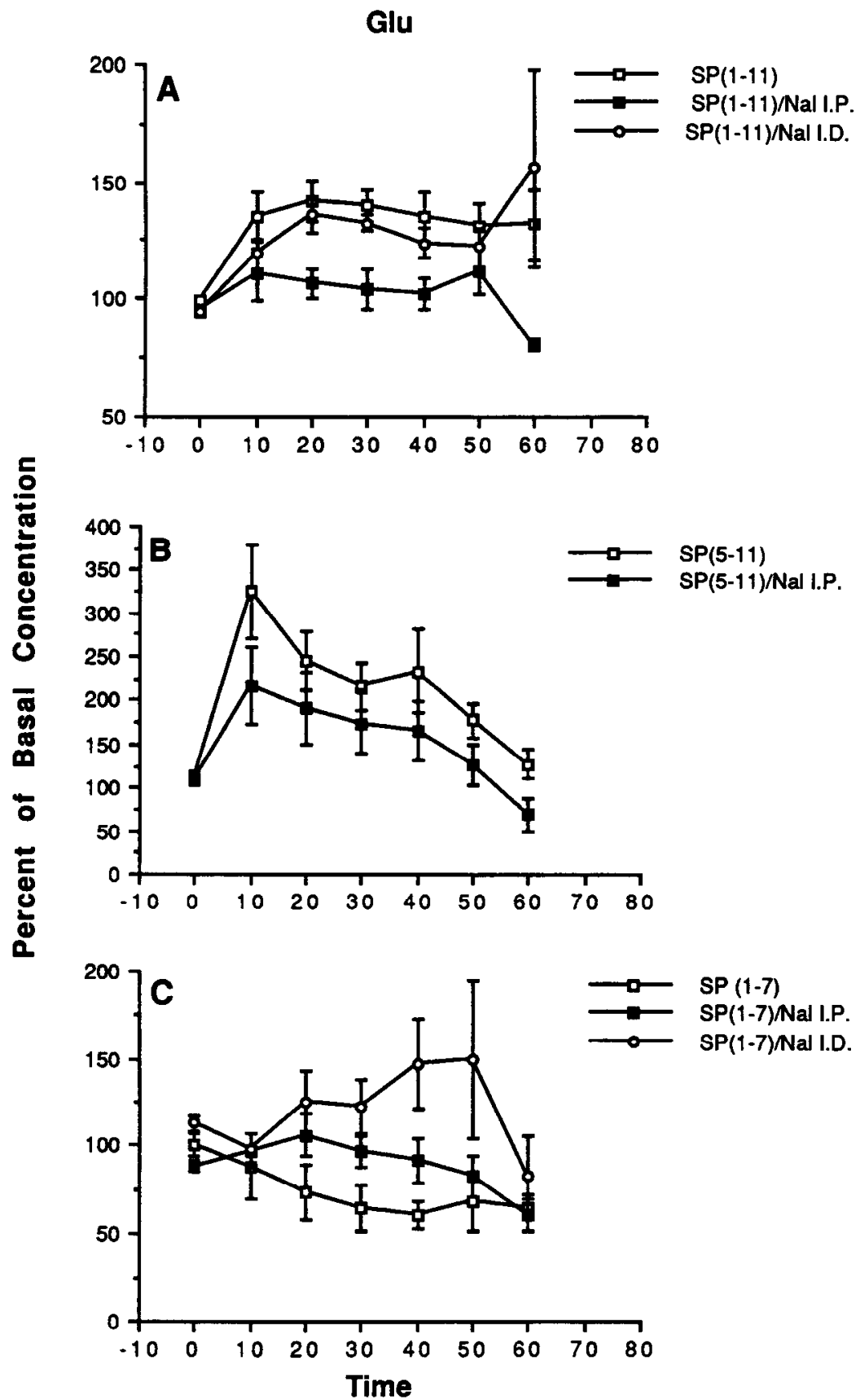

Figure 2. Time course of the changes in the concentration of Glu. See Figure 1 for details.

One possible mechanism for the analgesic effect of $\mathrm{SP}(1-7)$ is inhibition of $\operatorname{SP}(1-11)$ release. Morphine inhibits the release of $\mathrm{SP}(1-11)$ from the spinal cord in response to potassium or mechanical stimulation in vitro and in response to noxious stimulation in vivo (Yaksh et al., 1980; Hirota et al., 1985; Pang and Vasko, 1986). Hirota also observed inhibition of SP(1-11) release following met-enkephalin but not dynorphin, suggesting that a mu or delta opiate receptor is responsible for the morphine inhibition. The receptor for this morphine effect may be the same mu-1 receptor that $\mathrm{SP}(1-7)$ interacts with in binding studies, suggesting that $\mathrm{SP}(1-7)$ could act at the same receptor as morphine to inhibit $\mathrm{SP}(1-11)$ release.

The decrease in the basal release of Glu following SP(1-7) may involve presynaptic inhibition of Glu release at primary afferent terminals and this may also be a mechanism for the analgesic effect of SP(1-7). In contrast to Glu, Asp concentrations were not decreased following $\mathrm{SP}(1-7)$. One possible explanation for the inability to detect an effect of SP(1-7) on Asp is that basal concentrations of Asp, which are substantially lower than those of Glu, may reflect primarily metabolic rather than neuronal release.

In addition to inhibiting the SP(1-7)-induced decreases in amino acids, naloxone also inhibited the increases in amino acids following SP(1-11). This antagonism occurred only following systemic application of naloxone and not following coinfusion of SP(1-11) with naloxone, suggesting a supraspinal site of action distinct from that which inhibits the SP(1-7) effects. The ability of naloxone to inhibit both SP(1-11) increases and 


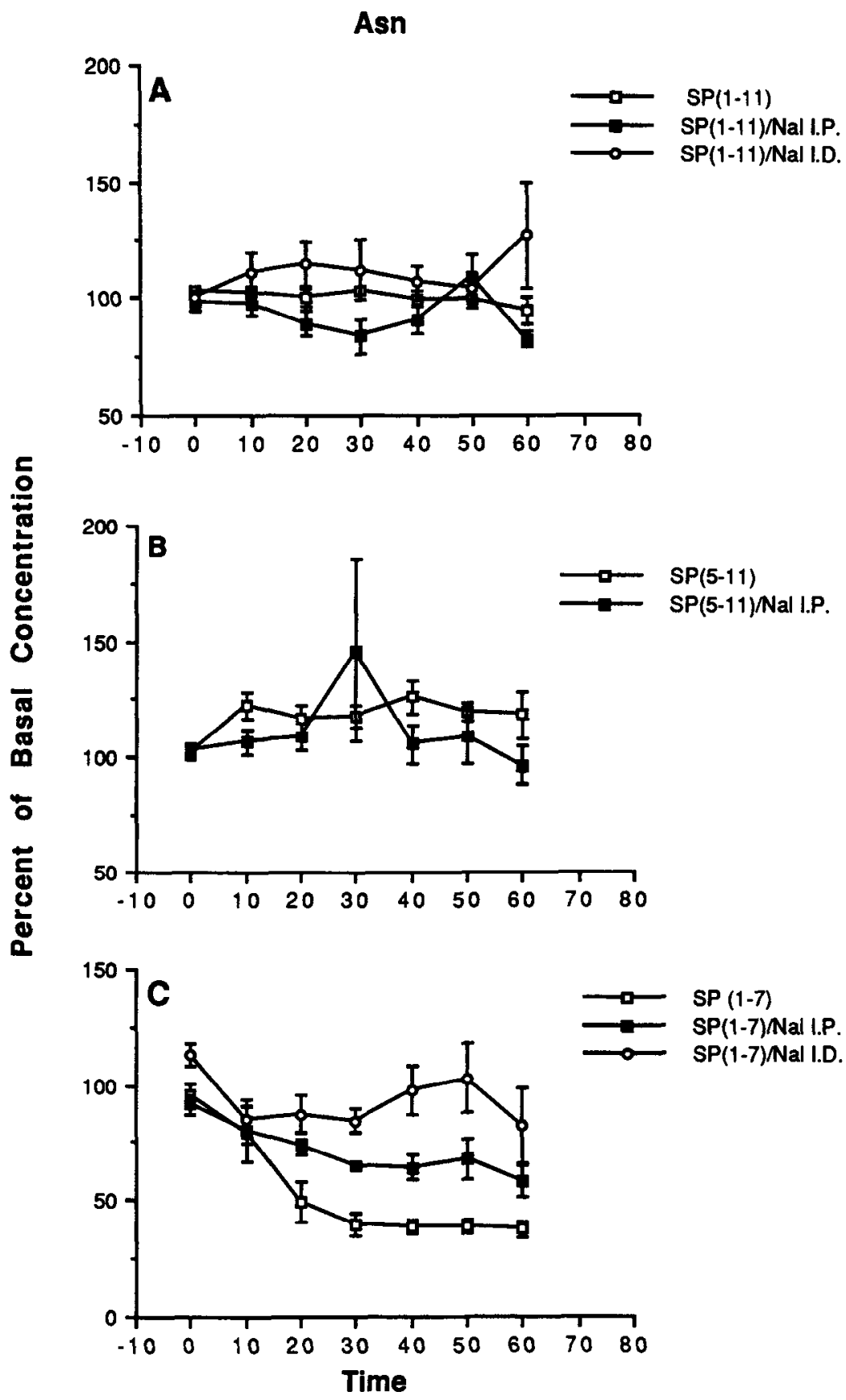

Figure 3. Time course of the changes in the concentration of Asn. See Figure 1 for details.

SP(1-7) decreases may explain the observation by Takahasi et al. (1987) that low doses of naloxone increase the biting and scratching produced by SP(1-11), possibly through inhibition of $\mathrm{SP}(1-7)$ acting at mu-1 receptors, and high doses of naloxone decrease the biting and scratching produced by SP(1-11), possibly through the same supraspinal opiate receptor that inhibits SP(1-11)-induced amino acid changes. Systemic naloxone did not inhibit the changes in amino acids produced by SP(5-11) application. The explanation for this SP(5-11) insensitivity to naloxone is not readily apparent but may relate to differences in receptor affinity or to differences in efficacy between $\operatorname{SP}(1$ $11)$ and $S P(5-11)$. It is also possible that naloxone sensitivity depends on simultaneous activation of $\mathrm{N}$ - and C-terminal SP receptors.
In contrast to $\mathrm{SP}(1-7), \mathrm{SP}(5-11)$ produced changes in amino acid release qualitatively similar to those seen following either formalin injection or SP(1-11) infusion, suggesting that the nociceptive effect of SP(1-11) involves activation of C-terminal receptors. This is in agreement with previous behavioral studies showing that intrathecal administration of C-terminal fragments of SP produce a caudally directed biting and scratching response that is not observed following injections of $\mathrm{N}$-terminal fragments (Igwe et al., 1988b). Activation of the C-terminal SP receptor appears to be primarily responsible for the excitatory effects observed following iontophoretic application of SP(1-11) onto dorsal horn neurons. Iontophoretic application of $\mathrm{N}$-terminal fragments shows only a weak scratching response compared to the response seen with SP or C-terminal fragments and 


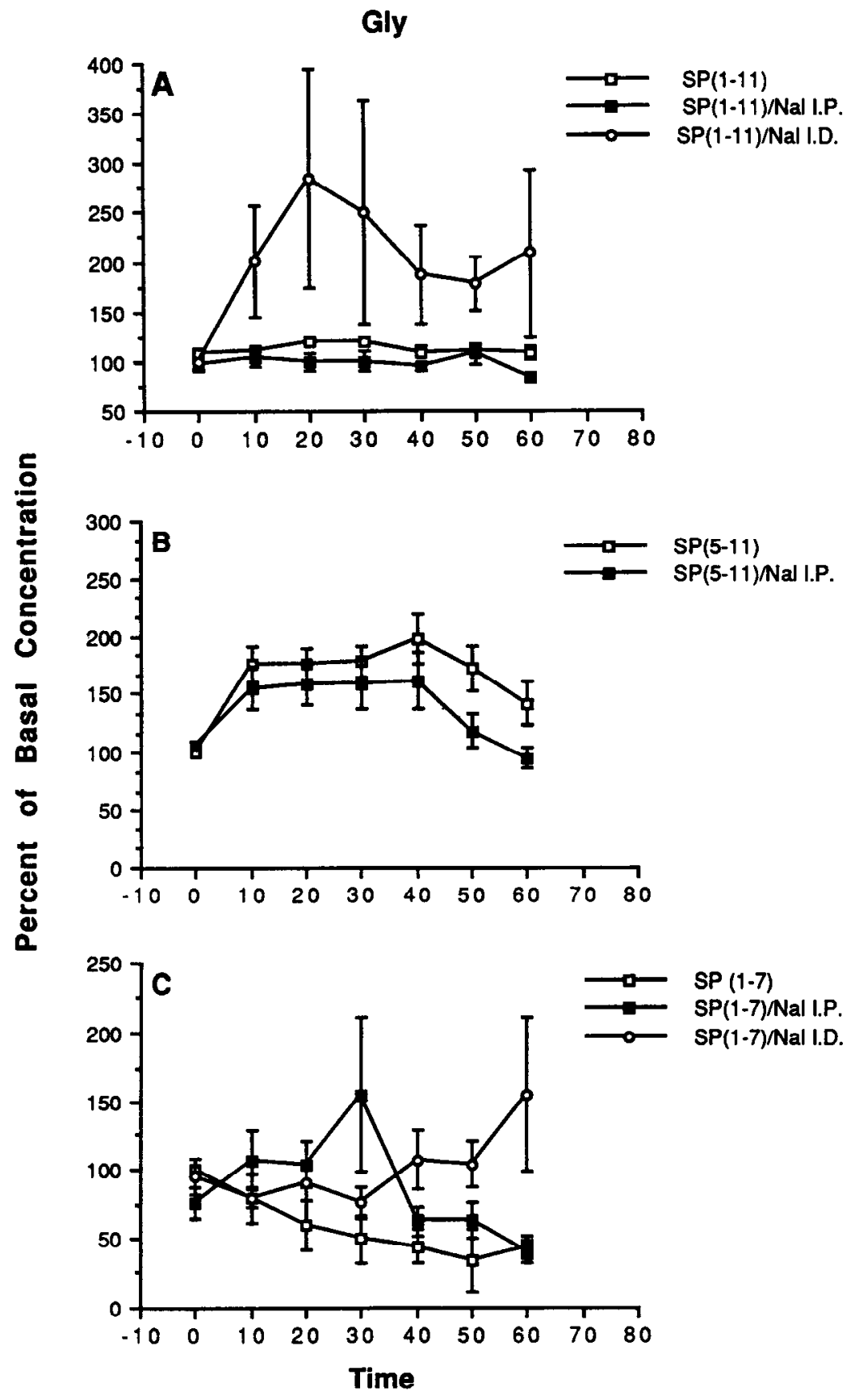

Figure 4. Time course of the changes in the concentration of Gly. See Figure 1 for details. then only at doses 1000 times greater than the effective dose of SP or C-terminal fragments (Piercey et al., 1982). The failure of naloxone to alter the C-terminal release of amino acids argues against activation of endogenous opiate systems by $\operatorname{SP}(1-11)$ as the mechanism for these changes.

Opposing actions of $\mathrm{N}$ - vs. C-terminal fragments of SP have previously been reported in many models (Bar-Shavit et al., 1980; Mazurek et al., 1981; Hall and Stewart, 1983, 1984; Gaffori et al., 1984; Hall et al., 1987a, b; Sakurada et al., 1988). The effects of $\mathrm{N}$ - and C-terminal fragments, however, are not always in opposition. Both $\mathrm{N}$ - and C-terminal fragments of SP, like SP itself, cause salivation in the rat and induce scratching following intracerebral-ventricular injection in mice (Piercey et al., 1982).
Aspartate has been proposed as a neurotransmitter in the CNS and recent immunohistochemical evidence suggests that $15 \%$ of unmyelinated axons in rat dorsal roots contain Asp-like immunoreactivity (Westlund et al., 1989). In our previous studies we have shown that release of Asp into the dorsal horn ECF following nociceptive stimulation is blocked by TTX, suggesting that the opening of voltage gated sodium channels is neccesary for this effect. This would be consistent with release of Asp from neuronal tissue but does not entirely rule out release from glial cells. In contrast to the effects following nociceptive stimulation, SP(1-11)-induced release of Asp was found in the present study to be TTX-insensitive. This suggests that if release was of neuronal origin, then $\operatorname{SP}(1-11)$ must act presynaptically. These results are also consistent with the hypothesis that nociceptive- 
Tau

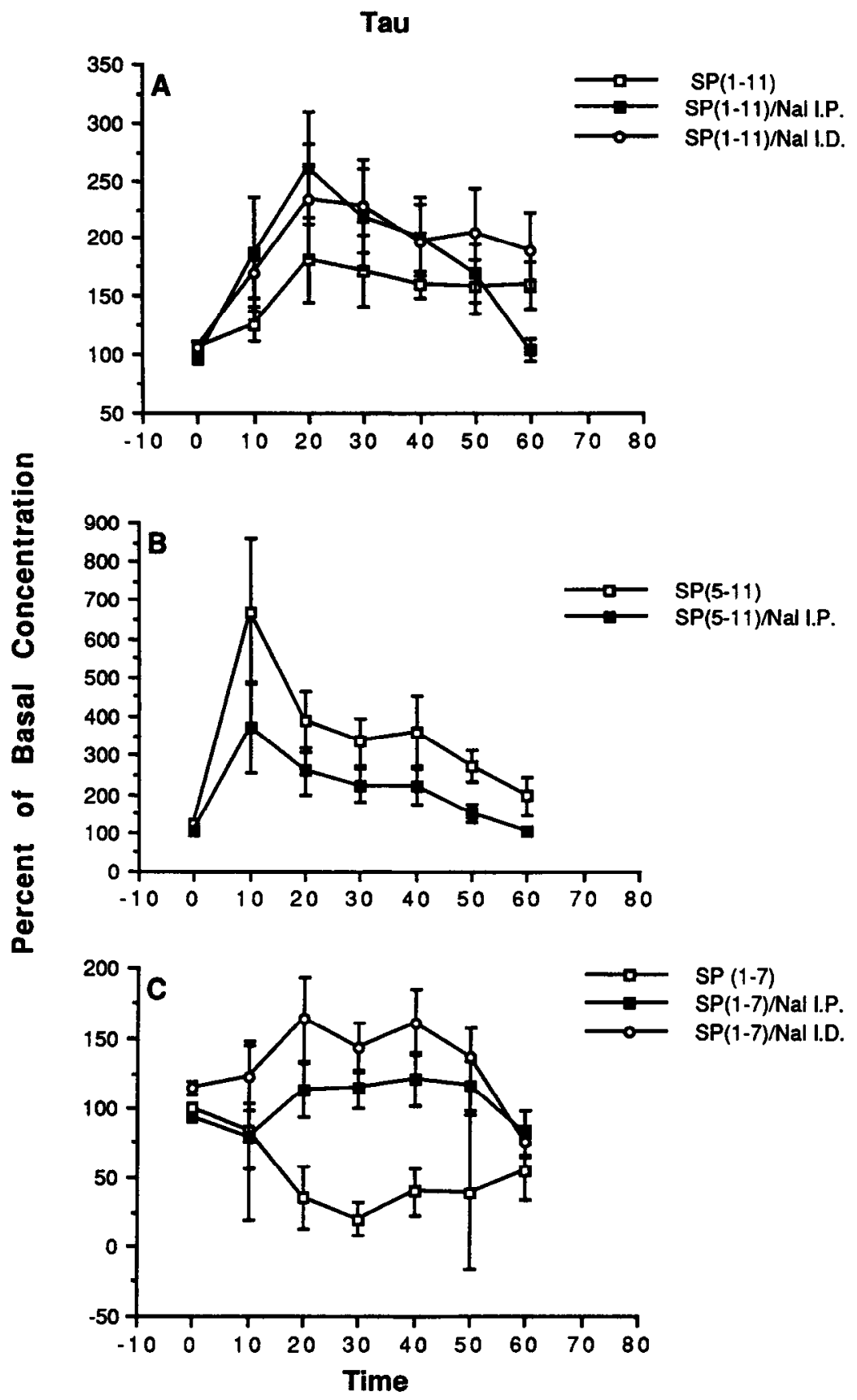

Figure 5. Time course of the changes in the concentration of Tau. See Figure 1 for details.

induced release of Asp is mediated by an initial release of SP(111). Release of Asp by SP(1-11) may, however, be distinct from nociceptive-induced release, as there are descending fibers and interneurons that also contain SP(1-11) but have projections to presynaptic sites in the spinal cord that may not be activated during nociceptive stimulation. Exogenous application of SP(111) may activate areas innervated by these SP fibers in addition to activating nociceptive pathways (Besson and Chaouch, 1987). This issue cannot be resolved from the present data.

We previously found that formalin also significantly increased the release of Glu into the dorsal spinal cord; however, there was considerable variability in the magnitude of this response. This variability was even greater when animals were pretreated with TTX. As a result, in the presence of TTX, formalin-stimulated Glu release was not significantly increased compared to basal concentrations but was also not significantly different from formalin-stimulated release in the absence of TTX. Therefore, it is not clear whether Glu release in response to formalin is sensitive to the blockade of sodium channels, as would be expected if release was neuronally mediated. It is also possible, however, that the lack of a significant effect of TTX on the formalin-induced release of Glu could reflect an inability of our model to distinguish between simultaneous metabolic and neuronal changes in Glu following physiological stimulation or could reflect the need to activate fewer sodium channels to initiate Glu release as compared to Asp release.

In distinct contrast to formalin-induced Glu release, $\mathbf{S P}(1$ 11)-induced release of Glu was substantially greater and was not only insensitive to TTX, but was enhanced by this pretreatment. If the release of Glu by SP(1-11) is of neuronal origin, 
then the failure of TTX to block release suggests that the site of this SP(1-11) effect is presynaptic. Given this, however, we would have predicted inhibition of formalin-induced release of Glu by TTX. As previously discussed, it is unclear whether this occurs. Alternatively, the failure of TTX to block Glu release could also support the hypothesis that SP(1-11)-induced Glu release is in part due to inhibition of Glu reuptake or release from nonneuronal tissue. The former hypothesis is supported by the observation that iontophoretic pretreatment of dorsal horn cells with SP(1-11), followed by a challenge with Glu when all SP-evoked activity has ceased, results in a prolonged potentiation of the effects of Glu (Puil, 1981). None of these hypotheses explain the enhanced release of Glu in the presence of TTX, but this effect could be due to TTX inhibition of sodium-channel-dependent release of other neurotransmitters involved in the inhibition of Glu release or enhancement of its uptake.

We previously reported that, in contrast to nociceptive stimulation, $\mathbf{S P}(1-11)$ induces a significant increase in the apparent release of the inhibitory amino acids Gly and Tau as well as Asp and Glu (Smullin et al., 1990). Release due to tissue damage was unlikely as concentrations of these amino acids returned to pretreatment levels within 10 min of discontinuation of SP(111) infusion. In the present study, pretreatment with TTX had no effect on the release of Gly, again suggesting either presynaptic or non-neuronal release. Taurine release, like Glu release, was enhanced by TTX, again suggesting a possible loss of neurotransmitters that cither inhibit the relcasc (metabolic or ncuronal) or enhance the uptake of Tau. In the present study, we observed an increase in Gly and Tau following SP(5-11) similar to that seen following SP(1-11), confirming the C-terminal involvement in this $\mathrm{SP}$ effect. In contrast, $\mathrm{SP}(1-7)$ significantly decreased the concentrations of Gly and Tau.

The potential role of Tau in nociception is unclear. Unlike the observed increases in Tau in the spinal cord ECF following SP infusion, tissue concentrations of Tau in the hypothalamus and lower brain-stem nuclei have been shown to decrease following acute pain in rats (Palkovits et al., 1986). Tau may be involved in inhibition of SP effects as it selectively antagonized the biting and scratching induced by intrathecal SP injection and inhibited acetic-acid-induced writhing in mice (Smullin et al., 1990). The effect of Tau in both paradigms was blocked by prior administration of the taurine antagonist 6-aminoethyl-3methyl-4H-1,2,4-benzothiadiazine-1,1-dioxide (TAG). In contrast to these analgesic-like effects, Tau alone, at higher doses, initiates a writhing behavior similar to that seen following i.p. injection of acetic acid (Larson, 1989).

The role of Gly in nociception is equally ambiguous, as Beyer et al. (1985) have reported hyperalgesia to cutaneous stimulation and a decrease in vocalization thresholds to tail shock following intrathecal injection of both Gly and the Gly antagonist strychnine. In mice, Gly only partially antagonizes SP-induced biting and scratching at a dose 10-fold greater than the effective dose of Tau (Smullin et al., 1990). One nociceptive role of Gly, however, may be related to its ability to potentiate the excitatory effects of Glu or Asp at the $N$-methyl-D-aspartate excitatory amino acid receptor subtype (Larson and Beitz, 1988). Decreases in Gly following SP(1-7) could result in partial loss of this receptor potentiation and account, in part, for the analgesic effects of SP(1-7).

The time course of the amino acid changes following $\mathrm{N}$ - and $\mathrm{C}$-terminal fragments were substantially different. The continuous decrease in amino acids following $\operatorname{SP}(1-7)$ is consistent with a gradual accumulation of the peptide within the extracellular fluid. An active uptake mechanism for SP(5-11) has been reported and activation of this system may be in part responsible for the reduction in the effects of SP(5-11) observed following the initial response (Nakata et al., 1981). While attenuation of the SP(5-11) effect on amino acids could also be due to either depletion of the neurotransmitter pool or inactivation or internalization of $\mathrm{NK}-1$ receptors, this explanation seems unlikely as no attenuation was seen following continuous SP infusion.

In conclusion, $\mathrm{N}$ - and $\mathrm{C}$-terminal metabolites of SP produce opposite effects on the apparent release of both excitatory and inhibitory amino acids from the dorsal spinal cord of rats. The C-terminal SP fragment, like SP itself, increases the apparent release of amino acids in a pattern similar to that following noxious stimulation and may therefore play a role in nociception. In contrast, the $\mathrm{N}$-terminal SP fragment produces a naloxone-sensitive decrease in amino acid release, supporting an interaction between $\mathrm{N}$-terminal SP metabolites, mu- 1 opiate receptors, and SP-induced analgesia. These $\mathrm{N}$-terminal-induced decreases may also play a role in desensitization to SP-induced motor activity in the spinal cord. In addition, studies with TTX support the hypothesis that nociception-induced Asp but not Glu release may be mediated by an initial release of SP(1-11). The enhanced release of Glu and Tau by $S P(1-11)$ in the presence of TTX suggests modulation of their release by other neurotransmitters.

\section{References}

Bar-Shavit, Z., R. Goldman, Y. Stabinsky, P. Gottlieb, M. Fridkin, V. I. Teichberg, and S. Blumberg (1980) Enhancement of phagocytosis-A newly found activity of substance $P$ residing in its N-terminal tetrapeptide sequence. Biochem. Biophys. Res. Commun. 94: 14451451.

Battaglia, G., and A. Rustioni (1988) Co-existence of glutamate and substance $P$ in dorsal root ganglion neurons of the rat and monkey. J. Comp. Neurol. 277: 302-312.

Besson, J., and A. Chaouch (1987) Peripheral and spinal mechanisms of nociception. Physiol. Rev. 67: 67-180.

Beyer, C., L. A. Roberts, and B. R. Komisaruk (1985) Hyperalgesia induced by altered glycinergic activity at the spinal cord. Life Sci. 37: 875-882.

Cridland, R. A., and J. L. Henry (1988) N-and C-terminal fragments of substance P: Spinal effects in the rat tail flick test. Brain Res. Bull. 20(4): 429-432.

Frederickson, R. C. A., V. Burgis, C. E. Harrel, and J. D. Edwards (1978) Dual actions of substance P on nociception: Possible role of endogenous opioids. Science 199: 1359-1362.

Gaffori, O., J. M. Stewart, and D. de Wied (1984) Influence of substance $\mathbf{P}$ and fragments on passive avoidance behavior. Experientia 40: 89-91.

Hall, M. E., and J. M. Stewart (1983) Substance P and behavior: Opposite effects of $\mathrm{N}$-terminal and C-terminal fragments. Peptides 4(5): 763-768.

Hall, M. E., and J. M. Stewart (1984) Modulation of isolation-induced fighting by $\mathrm{N}$ - and $\mathrm{C}$-terminal analogs of substance $\mathrm{P}$ : Evidence for multiplc recognition sites. Peptides 5(1): 85-89.

Hall, M. E., P. Grantham, J. Limoli, and J. M. Stewart (1987a) Effects of substance $P$ and neurokinin $A$ (substance $K$ ) on motor behavior: Unique effect of substance $P$ attributable to its amino-terminal sequence. Brain Res. 420: 82-94.

Hall, M. E., F. B. Miley, and J. M. Stewart (1987b) Modulation of blood pressure by substance P: Opposite effects of $\mathrm{N}$ - and C-terminal fragments on anesthetized rats. Life Sci. 40: 1909-1914.

Hayes, A. G., and M. B. Tyers (1979) Effects of intrathecal and intracerebroventricular injections of substance $P$ on nociception in the rat and mouse. Proc. of the B. P. S. 4th-6th: 488P.

Henry, J. L. (1976) Effects of substance $P$ on functionally identified units in cat spinal cord. Brain Res. 114: 439-451. 
Hirota, N., Y. Kuraishi, Y. Sato, M. Satoh, and H. Takagi (1985) Metenkephalin and morphine but not dynorphin inhibit noxious stimuliinduced release of substance $P$ from rabbit dorsal horn in situ. Neuropharmacology 24(6): 567-570.

Igwe, O. J., L. J. Felice, V. S. Seybold, and A. A. Larson (1988a) Optimization of high-performance liquid chromatography-radioimmunoassay protocols for the analyses of substance $P$ and some of its metabolic fragments. J. Chromatogr. 432: 113-126.

Igwe, O. J., X. Sun, and A. A. Larson (1988b) Role of Substance P (SP) metabolism in SP-induced desensitization in mice. Soc. Neurosci. Abstr. 14: 709.

Kawagoe, R., K. Onodera, and A. Takeuchi (1985) Release of endogenous glutamate from the frog spinal cord following dorsal root stimulation. Biomed. Res. 6: 239-245

Kawagoe, R., K. Onodera, and A. Takeuchi (1986) The release of endogenous glutamate from the newborn rat spinal cord induced by dorsal root stimulation and substance P. Biomed. Res. 7(4): 253-259.

Krummins, S. A., V. S. Seybold, and A. A. Larson (1989) Effects of substance $P$ (SP) and SP fragments on opioid receptor binding in mouse and spinal cord: Interaction of the $\mathrm{N}$-terminal portion of SP with $u_{1}$ sites. Adv. Biosci. (in press).

Larson, A. A. (1988) Desensitization to intrathecal substance $P$ in mice: Possible involvement of opioids. Pain 32: 367-374.

Larson, A. A. (1989) Intrathecal GABA, glycine taurine or beta-alanine elicits dyskinetic movements in mice. Pharmacol. Biochem. Behav. (in press).

Larson, A. A., and A. J. Beitz (1988) Glycine potentiates strychnineinduced convulsions: Role of NMDA receptors. J. Neurosci. $8(10)$ : $3822-3826$.

Lee, C. M., B. E. B. Sandberg, M. R. Hanley, and L. L. Iversen (1981) Purification and characterization of a membrane-bound substance P-degrading enzyme from human brain. Eur. J. Biochem 114: 315327.

Mazurek, N., I. Pecht, V. I. Teichberg, and S. Blumberg (1981) The role of the $\mathrm{N}$-terminal tetrapeptide in the histamine releasing action of substance P. Neuropharmacology 20: 1025-1027.

Nakata, Y., Y. Kusaka, H. Yajima, and T. Segawa (1981) Active uptake of substance $P$ carboxy-terminal heptapeptide (5-11) into rat brain and rabbit spinal cord slices. J. Neurochem. 37: 1529-1534.

Nyberg, F., P. Le Grevés, S. Sundqvist, and L. Terenius (1984) Characterization of substance $P(1-7)$ and (1-8) generating enzyme in human cerebrospinal fluid. Biochem. Biophys. Res. Commun. 125: 244-250.

Oehme, P., H. Hilse, E. Morgenstern, and E. Göres (1980) Substance P: Does it produce analgesia or hyperalgesia? Science 208: 305-307.

Palkovits, M., I. Elekes, T. Láng, and A. Patthy (1986) Taurine levels in discrete brain nuclei of rats. J. Neurochem. 47: 1333-1335.
Pang, I., and M. R. Vasko (1986) Morphine and norepinephrine but not 5-hydroxytryptamine and $\gamma$-aminobutyric acid inhibit the potassium-stimulated release of substance $P$ from rat spinal cord slices. Brain Res. 376: 268-279.

Piercey, M. F., P. J. K. Dobry, F. J. Einspahr, L. A. Schroeder, and N. Masiques (1982) Use of substance $P$ fragments to differentiate substance P receptors of different tissues. Reg. Peptides 3: 337-349.

Puil, E. (1981) S-glutamate: Its interactions with spinal neurons. Brain Res. 3: 229-322.

Sakurada, T., P. Le Grevés, J. Stewart, and L. Terenius (1985) Measurement of substance $P$ metabolites in rat CNS. J. Neurochem. 44: 718-722.

Sakurada, T., H. Kuwahara, K. Takahashi, S. Sakurada, K. Kisara, and L. Terenius (1988) Substance $\mathrm{P}(1-7)$ antagonizes substance P-induced aversive behaviour in mice. Neurosci. Lett. 95: 281-285.

Schneider, S. P., and E. R. Perl (1985) Selective excitation of neurons in the mammalian spinal dorsal horn by aspartate and glutamate in vitro: Correlation with location and excitatory input. Brain Res. 360: 339-343.

Skilling, S. R., D. H. Smullin, and A. A. Larson (1988) Extracellular amino acid concentrations in the dorsal spinal cord of freely moving rats following veratridine and nociceptive stimulation. J. Neurochem. 51: $127-132$.

Smullin, D. H., S. R. Skilling, and A. A. Larson (1990) Interactions between substance $P$, calcitonin gene-related peptide, taurine, and excitatory amino acids in the spinal cord. Pain (in press).

Stewart, J. M., C. J. Getto, K. Neldner, E. B. Reeve, W. A. Krivoy, and E. Zimmermann (1976) Substance $\mathbf{P}$ and analgesia. Nature 262 784-785.

Stewart, J. M., M. E. Hall, J. Harkins, R. C. A. Frederickson, L. Terenius, T. Hökfelt, and W. A. Krivoy (1982) A fragment of substance $P$ with specific central activity: SP(1-7). Peptides 3: 851-857.

Sweeney, M. I., and J. Sawynok (1986) Evidence that substance P may be a modulator rather than a transmitter of noxious mechanical stimulation. Can. J. Physiol. Pharmacol. 64: 1324-1327.

Takahasi, K., T. Sakurada, S. Sakurada, H. Kuwahara, A. Yonezawa, R. Ando, and K. Kisara (1987) Behavioural characterization of substance $\mathrm{P}$-induced nociceptive response in mice. Neuropharmacology 26(9): 1289-1293.

Westlund, K. N., D. L. McNeill, J. T. Patterson, and R. E. Coggeshall (1989) Aspartate immunoreactive axons in normal rat $\mathrm{L}_{4}$ dorsal roots. Brain Res. 489: 347-351.

Yaksh, T. L., T. M. Jessell, R. Gamse, A. W. Mudge, and S. E. Leeman (1980) Intrathecal morphine inhibits substance $P$ release from mammalian spinal cord in vivo. Nature 286: 155-157. 